\title{
Charge-memory polaron effect in molecular junctions
}

\author{
Dmitry A. Ryndyk, ${ }^{1}$ Pino D’Amico, ${ }^{1}$ Gianaurelio Cuniberti, ${ }^{2}$ and Klaus Richter ${ }^{1}$ \\ ${ }^{1}$ Institute for Theoretical Physics, University of Regensburg, D-93040 Regensburg, Germany \\ ${ }^{2}$ Institute for Material Science and Max Bergmann Center of Biomaterials, Dresden University of Technology, \\ D-01062 Dresden, Germany
}

(Received 19 June 2008; published 8 August 2008)

\begin{abstract}
The charge-memory effect, bistability, and switching between charged and neutral states of a molecular junction, as observed in recent scanning-tunneling microscope (STM) experiments, is considered within a minimal polaron model. We show that in the case of strong electron-vibron interaction, the rate of spontaneous quantum switching between charged and neutral states is exponentially suppressed at zero bias voltage but can be tuned through a wide range of finite switching time scales upon changing the bias. We further find that, while junctions with symmetric voltage drop give rise to random switching at finite bias, asymmetric junctions exhibit hysteretic behavior, enabling controlled switching. Lifetimes and charge-voltage curves are calculated by the master-equation method for weak coupling to the leads and at stronger coupling by the equation-ofmotion method for nonequilibrium Green's functions.
\end{abstract}

DOI: 10.1103/PhysRevB.78.085409

PACS number(s): 85.65.+h, 73.23.-b, 73.40.Gk, 73.63.-b

\section{INTRODUCTION}

Memory effects and switching at the molecular scale are the focus of present experimental and theoretical studies within molecular electronics. ${ }^{1-7}$ Besides stochastic switching in single-molecule junctions, ${ }^{4}$ recent scanning-tunneling microscope (STM) experiments ${ }^{2,3}$ show multistability of neutral and charged states of single metallic atoms coupled to a metallic substrate through a thin insulating ionic film. The switching was performed by the application of a finite voltage to the STM tip and was explained by the large ionic polarizability of the film. ${ }^{2}$

The coupling of a charge to the displacement of ions in the film can be treated as an electron-vibron interaction. If the energy of the unoccupied electron level without electronvibron interaction is $\epsilon_{0}$, the occupied (charged) state of the interacting system will have the energy $\epsilon_{1}=\epsilon_{0}-\epsilon_{p}$, where $\epsilon_{p}$ is so-called polaron shift (or recombination energy). Neutral and charged (polaron) states correspond to the local minima of the potential energy surface and are metastable if the electron-vibron interaction is strong enough. Applying an external voltage, one can change the state of this bistable system, an effect that is accompanied by hysteretic chargevoltage and current-voltage curves.

It was suggested ${ }^{8,9}$ that bistability between charged and neutral states can be accounted for in a single-level model, where one electron level is coupled to one vibration (Fig. 1). It was also shown that quantum switching between these metastable states can limit their lifetime and even result in telegraph noise at finite voltage rather than in a controlled switching. ${ }^{10,11}$ In this paper we investigate the crossover between these two pictures, depending on the relation between the time scales of quantum switching and voltage sweep. More precisely, the switching time $\tau$ between the two states of interest should be compared with the characteristic time of the external voltage sweeping, $\tau_{s} \sim V(t) /[d V(t) / d t]$. For $\tau \gg \tau_{s}$, quantum switching can be neglected and hysteresis can be observed, while in the opposite limit, $\tau \ll \tau_{s}$, the averaging removes the hysteresis. We calculate the charge- voltage curves and describe the full crossover between two regimes. In the single-level approximation it is not necessary to include Coulomb interaction explicitly, though one can additionally incorporate charging effects in the multilevel case.

The problem can be solved clearly and transparently in the limit of weak coupling to the leads, when the master equation for sequential tunneling can be used. Thus we focus our major discussion on this limit. In addition, the other limit of intermediate coupling to the leads is considered briefly and confirms our conclusions about the role of the electrostatic asymmetry.

The paper is organized as follows: In Sec. II we introduce the model. In Sec. III we outline the master-equation method and present the results in the case of weak coupling to the leads. In Sec. IV the case of intermediate coupling to the leads is considered briefly and compared with the weakcoupling case, before we conclude in Sec. V.

\section{MODEL}

The Hamiltonian of the single-level polaron model is

$$
\begin{aligned}
\hat{H}= & \left(\epsilon_{0}+e \varphi_{0}\right) d^{\dagger} d+\omega_{0} a^{\dagger} a+\lambda\left(a^{\dagger}+a\right) d^{\dagger} d \\
& +\sum_{i k}\left[\left(\epsilon_{i k}+e \varphi_{i}\right) c_{i k}^{\dagger} c_{i k}+\left(V_{i k} c_{i k}^{\dagger} d+\text { H.c. }\right)\right],
\end{aligned}
$$

where the first three terms describe the free-electron state, the free vibron of frequency $\omega_{0}(\hbar=1)$, and the electronvibron interaction. The other terms are the Hamiltonian of the leads and the tunneling coupling $(i=L, R$ is the lead index; $k$ labels electron states). The bias voltage is introduced through the left and right electrical potentials $V=\varphi_{L}-\varphi_{R}$. The energy level $\epsilon_{0}$ in Eq. (1) can be shifted through the gate voltage $V_{G}$. We choose as reference energy $\epsilon_{0}=0$ for $V_{G}=0$ and assume a linear capacitive coupling, $\epsilon_{0}=\alpha \mathrm{eV}_{G}$, setting $\alpha=1$.

The electrical potential $\varphi_{0}$ plays an important role in transport at finite bias voltages. It describes the shift in the 


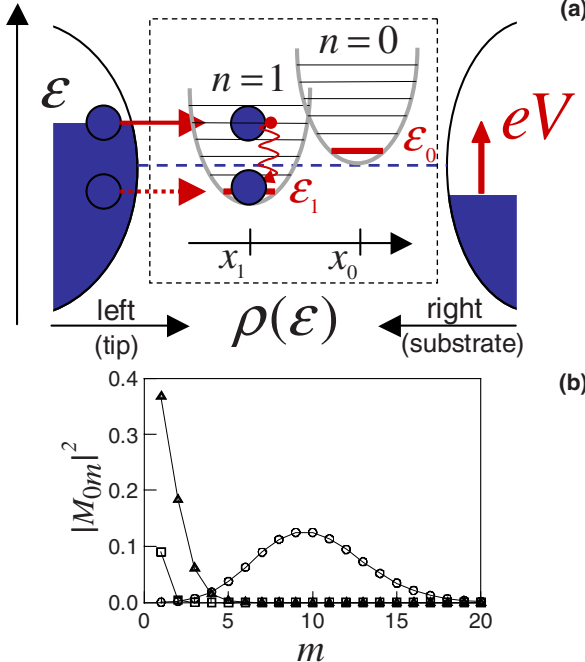

FIG. 1. (Color online) (a) The energy diagram of the single-level electron-vibron model, coupled to the left and right leads (or tip and substrate in the case of STM). (b) Franck-Condon matrix elements $M_{0 m}$ for weak ( $g=0.1$, squares), intermediate ( $g=1$, triangles), and strong $(g=10$, circles) interactions.

molecular level by the bias voltage, which is divided between the left lead (tip), the right lead (substrate), and the molecule as $\varphi_{0}=\varphi_{R}+\eta\left(\varphi_{L}-\varphi_{R}\right),{ }^{12}$ where $0<\eta<1$ describes the symmetry of the voltage drop across the junction: $\eta=0$ corresponds to the completely asymmetric case, while $\eta=0.5$ stands for the symmetric case. We assume the simplest linear dependence of the molecular potential ( $\eta=$ const), but its nonlinear dependence ${ }^{13}$ can be easily included in our model.

The coupling to the leads is characterized by the levelwidth function

$$
\Gamma_{i}(\epsilon)=2 \pi \sum_{k}\left|V_{i k}\right|^{2} \delta\left(\epsilon-\epsilon_{i k}\right) .
$$

In the wide-band limit considered below, the density of states in the leads $\rho(\epsilon)$ is constant and the coupling matrix elements $V_{i k}$ are assumed to be energy independent, so that $\Gamma_{L}$ and $\Gamma_{R}$ are constants. The full level broadening is given by the sum $\Gamma=\Gamma_{L}+\Gamma_{R}$.

Using the polaron (Lang-Firsov) ${ }^{14-16}$ canonical transformation, it is easy to show that the eigenstates of the isolated system $(\Gamma=0)$ are

$$
\left|\psi_{n m}\right\rangle=e^{-\lambda / \omega_{0}\left(a^{\dagger}-a\right) d^{\dagger} d}\left(d^{\dagger}\right)^{n} \frac{\left(a^{\dagger}\right)^{m}}{\sqrt{m !}}|0\rangle,
$$

with the eigenenergies

$$
E_{n m}=\epsilon_{1} n+\omega_{0} m, \quad \epsilon_{1}=\epsilon_{0}-\frac{\lambda^{2}}{\omega_{0}}, \quad \epsilon_{p}=\frac{\lambda^{2}}{\omega_{0}},
$$

where $n$ denotes the number of electrons, while the quantum number $m$ characterizes vibronic eigenstates, which are superpositions of states with different numbers of bare vibrons.

\section{WEAK COUPLING TO THE LEADS}

When the system is weakly coupled to the leads,

$$
\Gamma \ll \omega_{0}, \epsilon_{p},
$$

the polaron representation, Eqs. (3) and (4), is a convenient starting point. The qualitative picture of the sequential tunneling through a polaronic state is given in Fig. 1(a). Here the potential energies of the neutral and charged states are sketched as a function of the vibronic coordinate $x$. When an external voltage is applied, the energy levels are shifted depending on the asymmetry parameter $\eta$. It should be noted that this type of energy diagram is quite general for chargecontrolled bistable systems.

In the sequential-tunneling regime, the master equation for the probability $p_{n m}(t)$ of finding the system in one of the polaron eigenstates [Eq. (3)] can be written as ${ }^{17-20}$

$$
\frac{d p_{n m}}{d t}=\sum_{n^{\prime} m^{\prime}} \Gamma_{m m^{\prime}}^{n n^{\prime}} p_{n^{\prime} m^{\prime}}-\sum_{n^{\prime} m^{\prime}} \Gamma_{m^{\prime} m}^{n^{\prime} n} p_{n m}+I^{V}[p] .
$$

Here the first term describes the tunneling transition into the state $|n, m\rangle$ and the second term describes the transition out of the state $|n, m\rangle . I^{V}[p]$ is the vibron-scattering integral describing the relaxation of vibrons to equilibrium. The transition rates $\Gamma_{m m^{\prime}}^{n n^{\prime}}$ are found from the tunneling Hamiltonian [the last term in Eq. (1)]. Taking into account all possible single-electron-tunneling processes, we obtain the incoming and outgoing tunneling rates at zero bias voltage as

$$
\begin{gathered}
\Gamma_{m m^{\prime}}^{10}=\sum_{i=L, R} \Gamma_{i}\left(E_{1 m}-E_{0 m^{\prime}}\right)\left|M_{m m^{\prime}}\right|^{2} f_{i}^{0}\left(E_{1 m}-E_{0 m^{\prime}}\right), \\
\Gamma_{m m^{\prime}}^{01}=\sum_{i=L, R} \Gamma_{i}\left(E_{1 m^{\prime}}-E_{0 m}\right)\left|M_{m m^{\prime}}\right|^{2}\left[1-f_{i}^{0}\left(E_{1 m^{\prime}}-E_{0 m}\right)\right] .
\end{gathered}
$$

Here $f^{0}(\epsilon)$ is the equilibrium Fermi function and

$$
M_{m m^{\prime}}=\left\langle 0\left|\frac{a^{m}}{\sqrt{m !}} \exp \left[\frac{\lambda}{\omega_{0}}\left(a^{\dagger}-a\right)\right] \frac{\left(a^{\dagger}\right)^{m^{\prime}}}{\sqrt{m^{\prime} !}}\right| 0\right\rangle
$$

is the Franck-Condon matrix element, which is symmetric in $m-m^{\prime}$ and can be calculated analytically. For $m<m^{\prime}$ it reads

$$
M_{m<m^{\prime}}=\sum_{l=0}^{m} \frac{(-g)^{l} \sqrt{m ! m^{\prime} !} e^{-g / 2} g^{\left(m^{\prime}-m\right) / 2}}{l !(m-l) !\left(l+m^{\prime}-m\right) !},
$$

where $g=\left(\lambda / \omega_{0}\right)^{2}$ is the Huang-Rhys factor. ${ }^{21}$

One characteristic feature of these matrix elements in transport is the Franck-Condon blockade: ${ }^{19,20,22}$ In the case of strong electron-vibron interaction, the tunneling with small changes in $m$ is suppressed exponentially, as illustrated in Fig. 1(b) for the matrix element $M_{0 m}=e^{-g / 2 \frac{g^{m / 2}}{\sqrt{m !}}}$. Hence only tunneling through high-energy states is possible, which is also suppressed at low bias voltage and low temperature.

Finally, the average charge is

$$
\langle n\rangle(t)=\sum_{m} p_{1 m},
$$




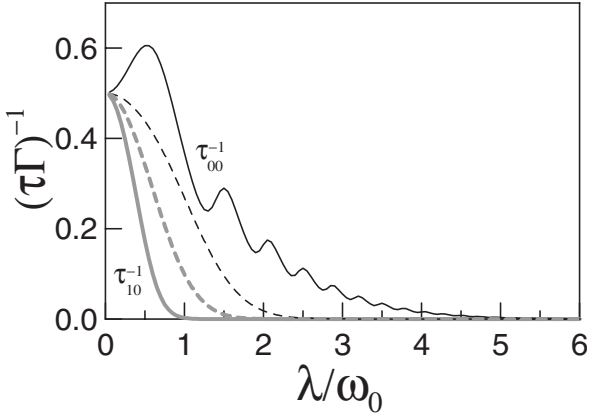

FIG. 2. Inverse lifetime $(\tau \Gamma)^{-1}$ of the neutral state (thin solid line) and the charged state (thick gray solid line) as a function of $\lambda / \omega_{0}$ at $\epsilon_{0}=\lambda^{2} / 2 \omega_{0}$ and the same at $\epsilon_{0}=0.9 \lambda^{2} / \omega_{0}$ (dashed lines); $k T=0.1 \omega_{0}$.

and the average current (from the left or the right lead) reads

$$
J_{i=L, R}(t)=e \sum_{m m^{\prime}}\left(\Gamma_{i m m^{\prime}}^{10} p_{0 m^{\prime}}-\Gamma_{i m m^{\prime}}^{01} p_{1 m^{\prime}}\right) .
$$

To proceed further, we calculate the characteristic lifetimes of the neutral and charged ground states. The lifetime $\tau_{n m}$ of the generic state $|n, m\rangle$ is given by the sum of the rates of all possible processes which change this state,

$$
\tau_{n m}^{-1}=\sum_{n^{\prime} m^{\prime}} \Gamma_{m^{\prime} m}^{n^{\prime} n} .
$$

As an example, calculating the lifetime of the neutral state $|0,0\rangle$, with an energy higher than the charged ground state $|1,0\rangle$, we find

$$
\tau_{00}^{-1}=\sum_{m} \sum_{i=L, R} \Gamma_{i}\left(E_{1 m}-E_{00}\right)\left|M_{m 0}\right|^{2} f_{i}^{0}\left(E_{1 m}-E_{00}\right) .
$$

For energy-independent $\Gamma_{i}$ (the wide-band limit), we obtain the simple analytical expression

$$
\tau_{00}^{-1}=\Gamma \sum_{m} e^{-g} \frac{g^{m}}{m !} f^{0}\left(\epsilon_{0}-\frac{\lambda^{2}}{\omega_{0}}+\omega_{0} m\right) .
$$

The corresponding expression for the lifetime of the charged state is (assuming that the equilibrium electrochemical potential in the leads is zero)

$$
\tau_{10}^{-1}=\Gamma \sum_{m} e^{-g} \frac{g^{m}}{m !} f^{0}\left(-\epsilon_{0}+\frac{\lambda^{2}}{\omega_{0}}+\omega_{0} m\right) .
$$

The dependence of tunneling rates (15) and (16) on the scaled electron-vibron interaction constant $\lambda / \omega_{0}$ is shown in Fig. 2. It is clearly seen that at large values of $\lambda$ the tunneling from the neutral state to the charged state and vice versa is exponentially suppressed in comparison with the bare tunneling rate $\Gamma$. Hence both states are (meta)stable at low temperatures and zero voltage.

Based on the experimental parameters of Ref. 2, the charged ground state is assumed to be below the equilibrium Fermi energy of the leads, while the neutral ground state is above it. In the experiments ${ }^{2}$ the observed relaxation energy

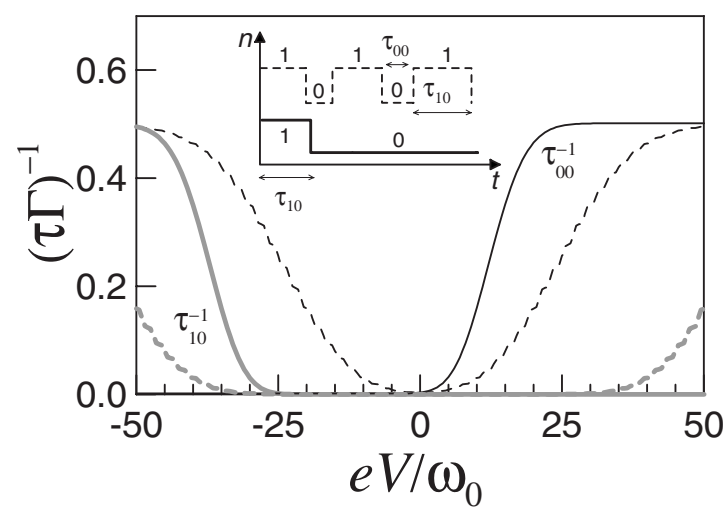

FIG. 3. Inverse lifetime $(\tau \Gamma)^{-1}$ as a function of normalized voltage $e V / \omega_{0}$ for the asymmetric junction $(\eta=0)$ at $\lambda / \omega_{0}=5$ and $\epsilon_{0}$ $=\lambda^{2} / 2 \omega_{0}$ for the neutral state (thin solid line) and the charged state (thick gray solid line) and the same for the symmetric junction ( $\eta=0.5$, dashed lines). Inset: random switching between bistable states (dashed line) and single switching into the stable state (full line) after a sudden change in the voltage.

$\epsilon_{p} \approx 2.4 \mathrm{eV}$ leads to the parameter $\lambda / \omega_{0}$ of order $5-10$. Thus the system is in the blockade regime at zero voltage; see Fig. 2.

Next we consider the other important question, whether a fast switching between the two states is possible. At finite voltage the switching rates are

$$
\begin{aligned}
\tau_{00}^{-1}= & \sum_{m} \frac{e^{-g} g^{m}}{m !}\left\{\Gamma_{L} f^{0}\left[\epsilon_{1}+\omega_{0} m-(1-\eta) e V\right]\right. \\
& \left.+\Gamma_{R} f^{0}\left(\epsilon_{1}+\omega_{0} m+\eta e V\right)\right\}, \\
\tau_{10}^{-1}= & \sum_{m} \frac{e^{-g} g^{m}}{m !}\left\{\Gamma_{L} f^{0}\left[-\epsilon_{1}+\omega_{0} m+(1-\eta) e V\right]\right. \\
& \left.+\Gamma_{R} f^{0}\left(-\epsilon_{1}+\omega_{0} m-\eta e V\right)\right\} .
\end{aligned}
$$

The voltage dependence of the inverse lifetime $(\tau \Gamma)^{-1}$ is shown in Fig. 3 for a junction with the same tunneling coupling $\Gamma_{L}=\Gamma_{R}$ but asymmetric voltage drop $(\eta=0)$, as well as for the completely symmetric junction $(\eta=0.5)$. The results in Fig. 3 imply that in both cases one can tune $(\tau \Gamma)^{-1}$ upon sweeping the bias voltage and thereby control the time scales for switching between charged and neutral states. For the symmetric junction both switching rates, $\tau_{00}^{-1}$ and $\tau_{10}^{-1}$ (dashed lines), are simultaneously nonzero at finite voltage $\left(\mathrm{eV} / \omega_{0}\right.$ $\geq 40$ for the parameters in Fig. 3), leading to random switching (noise), sketched as a dashed line in the inset. On the contrary, for the asymmetric junction, controlled switching into the neutral (black solid line) and charged (gray line) states can be achieved at large enough negative and positive voltages, respectively. This qualitatively different behavior is a result of the distinct voltage asymmetry of the two inverse lifetimes which are never both finite. The further peculiar feature of the asymmetric case, namely, that the switching rates of the neutral and charged states interchange their role as a function of the bias, i.e., the neutral (charged) state is long lived at negative (positive) bias, implies hysteretic behavior and a memory effect. 


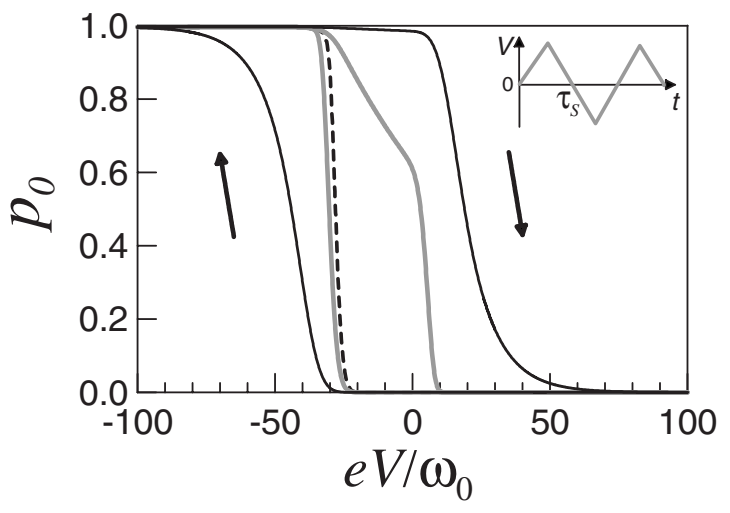

FIG. 4. Population of the neutral state as a function of normalized voltage $e V / \omega_{0}$ in the asymmetrical junction $(\eta=0)$ at $\lambda / \omega_{0}$ $=5$ and $\epsilon_{0}=\lambda^{2} / 2 \omega_{0}$ for fast voltage sweep (thin solid line), for slower sweep (thick gray solid line), and in the adiabatic limit (dashed line). Inset: sketch of voltage time dependence.

To this end we consider what happens if one sweeps the voltage with different velocities. At this point an assumption about the relaxation of vibrons without change in the charge state should be made. For simplicity we assume that the relaxation of vibrons is fast, $\tau_{V} \ll \tau, \tau_{s}$, so that after an electrontunneling event the system relaxes immediately into the vibronic ground state $|1,0\rangle$ or $|0,0\rangle$. In this case the probabilities $p_{1}$ of the charged state and $p_{0}$ of the neutral state are determined by the equations

$$
\begin{aligned}
& \frac{d p_{0}}{d t}=\tau_{10}^{-1} p_{1}-\tau_{00}^{-1} p_{0}, \\
& \frac{d p_{1}}{d t}=\tau_{00}^{-1} p_{0}-\tau_{10}^{-1} p_{1},
\end{aligned}
$$

with the lifetimes $\tau_{00}$ and $\tau_{10}$ from Eqs. (17) and (18).

Now let us consider the results for the asymmetric case $\eta=0$ (Fig. 4). If the voltage is changed fast enough, i.e., faster than the lifetime of charged and neutral states $\left(\tau \gg \tau_{s}\right.$ as discussed in Sec. I), then both states are stable at zero voltage (hysteresis). In the opposite (adiabatic) limit the voltage change is so slow that the system relaxes into the equilibrium state, and the population-voltage curve is single valued. Note that this controlled switching is possible only for asymmetric junctions for the reason given above.

\section{INTERMEDIATE COUPLING TO THE LEADS}

We finally compare the results with those of a further important limiting case, namely, the finite level width. Then the master-equation approach can no longer be used, and we apply alternatively the nonequilibrium Green's-function technique, ${ }^{23-30}$ which have been recently developed to treat vibronic effects in a self-consistent way. ${ }^{31-42}$ The average number of electrons is determined by the lesser Green's function $G^{<}\left(t_{1}-t_{2}\right)=i\left\langle d^{\dagger}\left(t_{2}\right) d\left(t_{1}\right)\right\rangle$ as

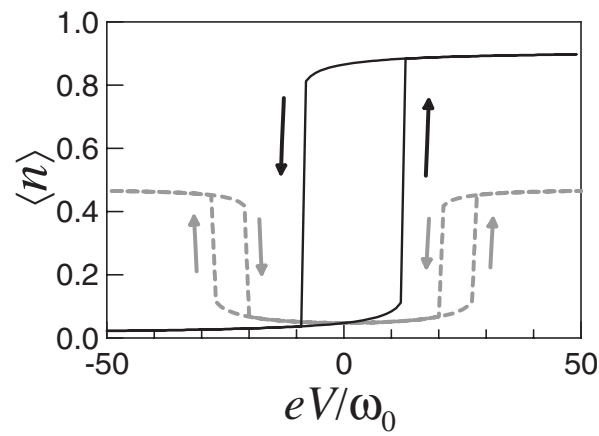

FIG. 5. Average number of electrons at $\Gamma_{L}=\Gamma_{R}=5 \omega_{0}$ as a function of normalized voltage $e V / \omega_{0}$ for the asymmetric junction, $\eta$ $=0$ (thin solid line), and for the symmetric junction, $\eta=0.5$ (dashed line), for $\lambda / \omega_{0}=5$ and $\epsilon_{0}=\lambda^{2} / \omega_{0}$.

$$
\langle n\rangle=-i \int G^{<}(\epsilon) \frac{d \epsilon}{2 \pi} .
$$

Actually, the lesser function $G^{<}(\epsilon)$ is used in the quantum kinetic formalism as a distribution function. In the singlelevel case the distribution function can be introduced through the relation

$$
G^{<}(\epsilon)=i A(\epsilon) f(\epsilon),
$$

where $A(\epsilon)=-2 \operatorname{Im} G^{R}(\epsilon)$ is the spectral density.

The calculation of Green's function is a nontrivial task even in the single-level model. It is simplified in the important limit of low vibron frequencies,

$$
\omega_{0} \ll \Gamma<\epsilon_{p},
$$

where the Born-Oppenheimer approximation holds true. We used the equation-of-motion approach in this case. In Fig. 5 the charge-voltage dependence, obtained in the simplest mean-field approximation, ${ }^{8,9}$ is shown. The spectral and the distribution functions in this case are given by the simple self-consistent expressions

$$
\begin{aligned}
& A(\epsilon)=\frac{2 \Gamma}{\left(\epsilon-\epsilon_{0}+\frac{2 \lambda^{2}}{\omega_{0}}\langle n\rangle-e \varphi_{0}\right)^{2}+\Gamma^{2}}, \\
& f(\epsilon)=\frac{\Gamma_{L} f_{L}^{0}\left(\epsilon-e \varphi_{L}\right)+\Gamma_{R} f_{R}^{0}\left(\epsilon-e \varphi_{R}\right)}{\Gamma_{L}+\Gamma_{R}} .
\end{aligned}
$$

The result is qualitatively the same as in the sequentialtunneling case: For electrically asymmetric junctions two stable states exist at zero bias (memory effect), which can be switched by the voltage. The current shows a similar hysteretic behavior as a function of voltage. For the symmetric junction, hysteresis is observed only at finite voltage (nonequilibrium bistability). Hence, asymmetric junctions are again preferable for a memory effect. We consider this limiting case in more detail in Ref. 43.

Finally we note that in the case $\omega_{0} \ll \Gamma$ we considered the stationary problem only, assuming that the switching rate between the two metastable states is small (compared, e.g., to $\Gamma$ ) at large $\lambda / \omega_{0}$. The calculation of the lifetimes of meta- 
stable states within the Green's-function approach and of dynamical effects arising from the competition between voltage sweeping and switching times, such as in Fig. 4, remains as a problem for the future.

\section{CONCLUSIONS}

To conclude, we considered a charge-memory effect and switching phenomena in single-molecule junctions in the framework of a simple polaron model, taking into account nonstationary effects, in particular the interplay between time scales of voltage sweeping and quantum switching rates from metastable states. We showed that the bistability arises if the quantum switching between neutral and charged states involved are suppressed, e.g., due to Franck-Condon blockade. In the quasiclassical language this means that there are two local minima of the energy and the barrier height between these two states is large enough.

In view of possible experimental realizations and applications of the memory effect, the lifetime of the memory states should be large compared with the other important time scale of the problem, namely, the voltage sweeping time. In this paper, employing the time-dependent master-equation method, different regimes, characterized by random mutual transitions and by single switching events into a stable configuration, are identified. In the latter case, controlled switching of the molecule is achieved by applying finite voltage pulses.

Special attention is paid to the role of the junction asymmetry and its influence on the memory effect. Electrostatically symmetric and asymmetric junctions show qualitatively different bistability behaviors. We found that in the case of asymmetric bias-voltage drop across the junction, $\eta=0$, both neutral and charged states can be unstable at one polarity of bias voltage and stable at the opposite polarity. Under an appropriate choice of parameters, the instability regions for the two memory states do not overlap. Thus a definite memory state can be obtained. Moreover both states are (meta)stable at zero bias voltage. Finally, the finite thresholds in bias voltage prevent accidental switching by noise or a weak external signal. These properties enable in principle a memory functionality of the system including writing of two states and readout by a small bias voltage. Note that such an asymmetric case is typically achieved in STM experiments.

In the case of symmetric voltage drop, $\eta=0.5$, the situation is different. At finite bias voltage the energy level is inside the transport window, between the left and the right electrochemical potentials. Consequently, the system is permanently switching between two states, and it is not possible to fix one definite memory state. The symmetric situation can also give rise to a hysteretic behavior but only at finite bias voltages, making this case less interesting for the memory effect addressed here.

\section{ACKNOWLEDGMENTS}

We acknowledge fruitful discussions with J. Repp. This work was funded by the Deutsche Forschungsgemeinschaft within the Priority Program No. SPP 1243, Collaborative Research Center SFB 689 (D.A.R.), and within the international collaboration "Single molecule based memories" under Grant No. CU 44/3-2 (D.A.R.).
${ }^{1}$ A. S. Alexandrov and A. M. Bratkovsky, Phys. Rev. B 67, 235312 (2003).

${ }^{2}$ J. Repp, G. Meyer, F. E. Olsson, and M. Persson, Science 305, 493 (2004).

${ }^{3}$ F. E. Olsson, S. Paavilainen, M. Persson, J. Repp, and G. Meyer, Phys. Rev. Lett. 98, 176803 (2007).

${ }^{4}$ E. Lörtscher, H. B. Weber, and H. Riel, Phys. Rev. Lett. 98, 176807 (2007).

${ }^{5}$ P. Liljeroth, J. Repp, and G. Meyer, Science 317, 1203 (2007).

${ }^{6} \mathrm{M}$. del Valle, R. Gutiérrez, C. Tejedor, and G. Cuniberti, Nat. Nanotechnol. 2, 176 (2007).

${ }^{7}$ G. Cuniberti, G. Fagas, and K. Richter, Introducing Molecular Electronics (Springer-Verlag, Berlin, 2005).

${ }^{8}$ A. C. Hewson and D. M. Newns, J. Phys. C 12, 1665 (1979).

${ }^{9}$ M. Galperin, M. A. Ratner, and A. Nitzan, Nano Lett. 5, 125 (2005).

${ }^{10}$ A. Mitra, I. Aleiner, and A. J. Millis, Phys. Rev. Lett. 94, 076404 (2005).

${ }^{11}$ D. Mozyrsky, M. B. Hastings, and I. Martin, Phys. Rev. B 73, 035104 (2006).

${ }^{12}$ S. Datta, W. Tian, S. Hong, R. Reifenberger, J. I. Henderson, and C. P. Kubiak, Phys. Rev. Lett. 79, 2530 (1997).

${ }^{13}$ T. Rakshit, G. C. Liang, A. W. Ghosh, M. Hersam, and S. Datta, Phys. Rev. B 72, 125305 (2005).
${ }^{14}$ I. G. Lang and Y. A. Firsov, Sov. Phys. JETP 16, 1301 (1963).

${ }^{15}$ A. C. Hewson and D. M. Newns, Jpn. J. Appl. Phys., Suppl. 2, 121 (1974)

${ }^{16}$ G. Mahan, Many-Particle Physics (Plenum, New York, 1990).

${ }^{17}$ S. Braig and K. Flensberg, Phys. Rev. B 68, 205324 (2003).

${ }^{18}$ A. Mitra, I. Aleiner, and A. J. Millis, Phys. Rev. B 69, 245302 (2004).

${ }^{19}$ J. Koch and F. von Oppen, Phys. Rev. Lett. 94, 206804 (2005).

${ }^{20}$ J. Koch, M. Semmelhack, F. von Oppen, and A. Nitzan, Phys. Rev. B 73, 155306 (2006).

${ }^{21}$ K. Huang and A. Rhys, Proc. R. Soc. London, Ser. A 204, 406 (1950).

${ }^{22}$ K. C. Nowack and M. R. Wegewijs, arXiv:cond-mat/0506552 (unpublished).

${ }^{23}$ L. Kadanoff and G. Baym, Quantum Statistical Mechanics (Benjamin, New York, 1962).

${ }^{24}$ L. V. Keldysh, Zh. Eksp. Teor. Fiz. 47, 1515 (1964); [Sov. Phys. JETP 20, 1018 (1965)].

${ }^{25}$ J. Rammer and H. Smith, Rev. Mod. Phys. 58, 323 (1986).

${ }^{26}$ Y. Meir and N. S. Wingreen, Phys. Rev. Lett. 68, 2512 (1992).

${ }^{27}$ A.-P. Jauho, N. S. Wingreen, and Y. Meir, Phys. Rev. B 50, 5528 (1994).

${ }^{28}$ H. Haug and A.-P. Jauho, Quantum Kinetics and Optics of Semiconductors, Springer Series in Solid-State Sciences Vol. 123 
(Springer, New York, 1996).

${ }^{29}$ A.-P. Jauho, J. Phys.: Conf. Ser. 35, 313 (2006).

${ }^{30}$ D. A. Ryndyk, R. Gutiérrez, B. Song, and G. Cuniberti, in Energy Flow Dynamics in Biomaterial Systems, edited by E. Bittner and I. Burghardt, Lecture Notes in Physics (Springer, Heidelberg, to be published); arXiv:0805.0628.

${ }^{31}$ P. Král, Phys. Rev. B 56, 7293 (1997).

${ }^{32}$ K. Flensberg, Phys. Rev. B 68, 205323 (2003).

${ }^{33}$ M. Galperin, M. A. Ratner, and A. Nitzan, Nano Lett. 4, 1605 (2004).

${ }^{34}$ M. Galperin, M. A. Ratner, and A. Nitzan, J. Phys. Chem. 121, 11965 (2004).

${ }^{35}$ T. Frederiksen, M.S. thesis, Technical University of Denmark, 2004.
${ }^{36}$ M. Hartung, M.S. thesis, University of Regensburg, 2004.

${ }^{37}$ T. Frederiksen, M. Brandbyge, N. Lorente, and A.-P. Jauho, Phys. Rev. Lett. 93, 256601 (2004).

${ }^{38}$ D. A. Ryndyk and J. Keller, Phys. Rev. B 71, 073305 (2005).

${ }^{39}$ M. Galperin, A. Nitzan, and M. A. Ratner, Phys. Rev. B 73, 045314 (2006).

${ }^{40}$ D. A. Ryndyk, M. Hartung, and G. Cuniberti, Phys. Rev. B 73, 045420 (2006).

${ }^{41}$ M. Galperin, M. A. Ratner, and A. Nitzan, J. Phys.: Condens. Matter 19, 103201 (2007).

${ }^{42}$ D. A. Ryndyk and G. Cuniberti, Phys. Rev. B 76, 155430 (2007).

${ }^{43}$ P. D'Amico, D. A. Ryndyk, G. Cuniberti, and K. Richter, New J. Phys. 10, 085002 (2008). 